The Astrophysical Journal, 295:L27-L31, 1985 August 15

(C) 1985. The American Astronomical Society. All rights reserved. Printed in U.S.A.

\title{
A NEW LUMINOSITY COMPONENT IN 3C 48
}

\author{
G. Neugebauer, ${ }^{1}$ B. T. Soifer, ${ }^{1}$ AND G. K. Miley ${ }^{2}$ \\ Received 1985 April 29; accepted 1985 May 23
}

\begin{abstract}
The infrared emission from the quasar $3 \mathrm{C} 48$ has a luminosity $L \approx 5 \times 10^{12} L_{\odot}$ and dominates the power output of the quasar system. It is shown that the infrared emission, unlike that of most radio-loud quasars, is most likely not an extension of the radio emission of the quasar. It is argued that the infrared emission in $3 \mathrm{C} 48$ is probably thermal radiation arising in a highly luminous galaxy surrounding the quasar.
\end{abstract}

Subject headings: infrared: sources - quasars

\section{INTRODUCTION}

The all-sky survey by the Infrared Astronomical Satellite (IRAS) from 12 to $100 \mu \mathrm{m}$ presented an opportunity to make unbiased observations of quasar continua to determine the importance of far-infrared radiation in the total luminosity of quasars. Although the sensitivity of the IRAS survey was such that only a few quasars were unambiguously detected, the survey did show the presence of unexpectedly strong emission from the well-known quasar $3 \mathrm{C} 48$. Among the radio-loud quasars, except for $3 \mathrm{C} 48$, the only quasars detected were those where the infrared emission could reasonably be attributed to an extension of the (flat) radio continuum. In contrast, 3C 48 did show unexpectedly strong far-infrared emission, well above that expected from a smooth extrapolation of the radio continuum to far-infrared wavelengths. The far-infrared emission, in fact, dominates the luminosity of that quasar.

\section{OBSERVATIONS}

Figure 1 shows the infrared spectral indices of 22 quasars detected in the IRAS survey with good quality fluxes (IRAS Supplement 1985). The spectral indices $\alpha$ are defined such that the flux density $f_{\nu}$ is proportional to $\nu^{\alpha}$, where $\nu$ is the frequency. The sample included all quasars from the catalog of Véron-Cetty and Véron (1983) who "arbitrarily defined a quasar as a starlike object, or object with a starlike nucleus, brighter than absolute (visual) magnitude - 23." In addition to the detections whose spectral indexes are shown in Figure 1 , there was an approximately equal number of $I R A S$ sources with positional coincidences with quasars, but which were either poor quality IRAS detections or which were masked or confused by the presence of a nearby galaxy.

Where pointed observations of the quasars found in the survey have been obtained, these measurements, which have significantly higher signal-to-noise ratios than it is possible to obtain from the survey itself, have been used in Figure 1 (Neugebauer et al. 1984). For six of the 22 objects, pointed

\footnotetext{
${ }^{1}$ Division of Physics, Mathematics and Astronomy, California Institute of Technology.

${ }^{2}$ Space Telescope Science Institute (on contract from European Space Agency) and Sterrewacht, Leiden, The Netherlands.
}

observations were not available and the measurements used to obtain the spectral indexes were gotten by co-addition of the survey scans at the location of the detected quasars in order to increase the signal-to-noise ratios. The uncertainties in the measurements of the flux densities are of the order of $10 \%$, leading to uncertainties of $0.2-0.3$ in the spectral indexes.

Figure 1 shows that the majority of the quasars lie close to the line of constant spectral index from 25 through $100 \mu \mathrm{m}$. The spectral indexes of a sample of galaxies with flux densities at $60 \mu \mathrm{m}>5 \mathrm{Jy}$ are included in Figure 1 (Soifer et al. 1985). In contrast to the quasars, the majority of galaxies found by IRAS populate fairly uniformly the area which is bounded by $-3<\alpha(100,60)<0$ and $-3<\alpha(60,25)<-1$. The largest deviations from the line of constant spectral index shown by quasars are exhibited by $3 \mathrm{C} 48$ and the two Markarian galaxies, Mrk 231 and Mrk 1014. These three sources lie in the area where spectral indexes are steeper in $\alpha(60,25)$ and flatter in $\alpha(100,60)$ than the line of constant spectral index, thus bringing them closer to the area occupied by the majority of galaxies. The latter two objects have also been identified as Seyfert 1 galaxies but are included in the sample because of the luminosity criterion applied by Véron-Cetty and Véron (1983).

Figure 2 shows the continuum energy distributions of $3 \mathrm{C} 48$ and Mrk 231. Although the ratio of the infrared-to-radio fluxes differs by a factor of 1000 between the two quasars, the gross similarity of the infrared continua is evident. For $3 \mathrm{C} 48$, the observed color temperature between 60 and $100 \mu \mathrm{m}$ is $T_{c}$ $(60,100)=55 \mathrm{~K}$, while that between 25 and $60 \mu \mathrm{m}$ is $T_{c}(25,60)=81 \mathrm{~K}$. The observations at 60 and $100 \mu \mathrm{m}$ imply an observed far-infrared luminosity for $3 \mathrm{C} 48$ of $5 \times 10^{12} L_{\odot}$ if the radiation is isotropic and the redshift, $z$, is cosmological. The Hubble constant has been taken as $60 \mathrm{~km} \mathrm{sec}^{-1} \mathrm{Mpc}^{-1}$, and the deceleration parameter $q_{0}$ has been assumed to be 0 ; these values will be used throughout this Letter.

In Figure 3, The energy distribution of $3 \mathrm{C} 48$ is compared to that of two well-known radio-loud quasars, 3C 273 and 3C 345 . These latter quasars are both known to be rapidly variable; their far-infrared emission is thought to be primarily an extension of the radio wavelength emission. It is clear from Figure 3 that the continuum distribution of $3 \mathrm{C} 48$, dominated by the peak at $60 \mu \mathrm{m}$, differs markedly from those of $3 \mathrm{C} 273$ 


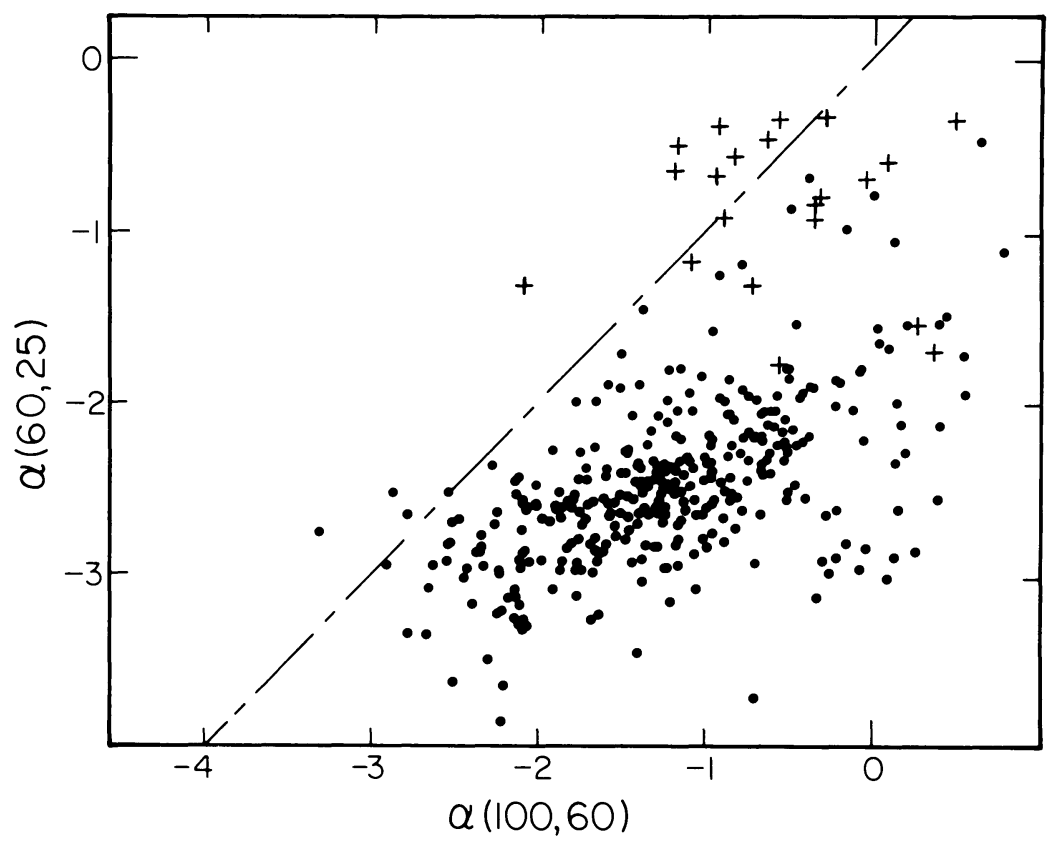

FIG. 1.-A plot is given of the spectral indexes between 25 and $60 \mu \mathrm{m}[\alpha(60,25)]$ and those between 60 and $100 \mu \mathrm{m}[\alpha(60,100)]$ for the quasars and bright infrared galaxies found in the IRAS survey. The spectral indexes and samples are defined in the text. Quasars are indicated by + while bright galaxies are indicated by $\bullet$. The dashed line represents equal spectral indexes. The uncertainty in each coordinate is about 0.3 . The three quasars with the lowest values of $\alpha(60,25)$ are 3C 48, Mrk 231, and Mrk 1014, with $\alpha(60,25) \approx-1.6$ and $\alpha(100,60) \approx 0$.

and $3 \mathrm{C}$ 345. The fourth quasar shown in Figure 3, PG $1351+640$, is an example of a radio-quiet quasar whose infrared emission also dominates its luminosity, but where the peak emission occurs at $25 \mu \mathrm{m}$.

\section{ORIGIN OF INFRARED LUMINOSITY}

Although the origin of the infrared luminosity cannot be decided with certainty, the infrared radiation is probably not an extension of the radio source. Although compact radio sources with dimensions around $0^{\prime \prime} 05$ are present in $3 \mathrm{C} 48$ (Wilkinson et al. 1984), there is no evidence of a submilliarcsecond, flat-spectrum radio source. Primarily there is no evidence for a violent variable radio source, such as seen in $3 \mathrm{C}$ 345 , which is generally indicative of a flat radio continuum. The near-infrared (1.3-2.2 $\mu \mathrm{m})$ emission of 3C 48 has been monitored for $15 \mathrm{yr}$ at the Palomar Observatory and, in contrast to $3 \mathrm{C} 345$, shows no evidence of significant variability. Thus $3 \mathrm{C} 48$ has little or no resemblance to those quasars, such as $3 C 273$ and $3 C$ 345, where there is strong evidence that the far-infrared continuum is an extension of the radio synchrotron spectrum.

A much more likely explanation for the origin of the infrared emission is that it comes from thermal reradiation of heated dust. If the radiation truly represents thermal emission and if the emissivity of dust grains is proportional to (wavelength $)^{-1}$, the representive temperature derived from the 60 and $100 \mu \mathrm{m}$ observations is $57 \mathrm{~K}$ in the rest system of $3 \mathrm{C} 48$, while that derived from the 25 and $60 \mu \mathrm{m}$ observations is 91 $\mathrm{K}$. The dispersion probably indicates that a range of tempera- tures is in reality present. If the grains are heated by a single luminosity source with luminosity $5 \times 10^{12} L_{\odot}$, the grains at $57 \mathrm{~K}$ must be $\sim 2 \mathrm{kpc}$ from the source; the actual distance depends on the detailed properties of the grain absorption.

An estimate of the mass of dust present depends on whether the dust is optically thin or thick. If the radiation is optically thin, the mass can be estimated from

$$
M=\frac{D^{2} f_{\nu}(1+z)}{\kappa B_{\nu_{0}}(T)},
$$

where $D$ is the distance to the quasar, $f_{v}$ is the observed flux density at the frequency $\nu_{0}, B_{\nu_{0}}(T)$ is the Planck blackbody function at a rest frequency $\nu_{0}$ and temperature $T$, and $\kappa$ is the mass absorption coefficient at $\nu_{0}$. If $\kappa=25 \mathrm{~m}^{2} \mathrm{~kg}^{-1}$ (Draine and Lee 1984), the mass derived from the 60 and 100 $\mu \mathrm{m}$ observations, i.e., that radiating at $57 \mathrm{~K}$, is $M_{d}=6 \times 10^{7}$ $M_{\odot}$. The warmer material (>90 K) radiating in the 25 to 60 $\mu \mathrm{m}$ region contributes only $7 \times 10^{6} M_{\odot}$ to the total mass.

The estimates of the mass of dust derived above imply, with any normal gas-to-dust mass ratio, a mass of gas significantly in excess of the mass of gas expected for either the broad or narrow emission-line regions of quasars. If the gas-to-dust ratio is $\sim 200$ in $3 \mathrm{C} 48$, then the gas associated with the emitting dust must be $>10^{10} M_{\odot}$. Baldwin (1975) estimates that typical broad-line regions in quasars contain $<10^{3} M_{\odot}$, while $10^{3}-10^{7} M_{\odot}$ exist in the narrow-line regions. It is well known that nebulosity, probably not to be associated directly with the host galaxy in 3C 48, extends asymmetrically $\sim 100$ 
No. 2,1985

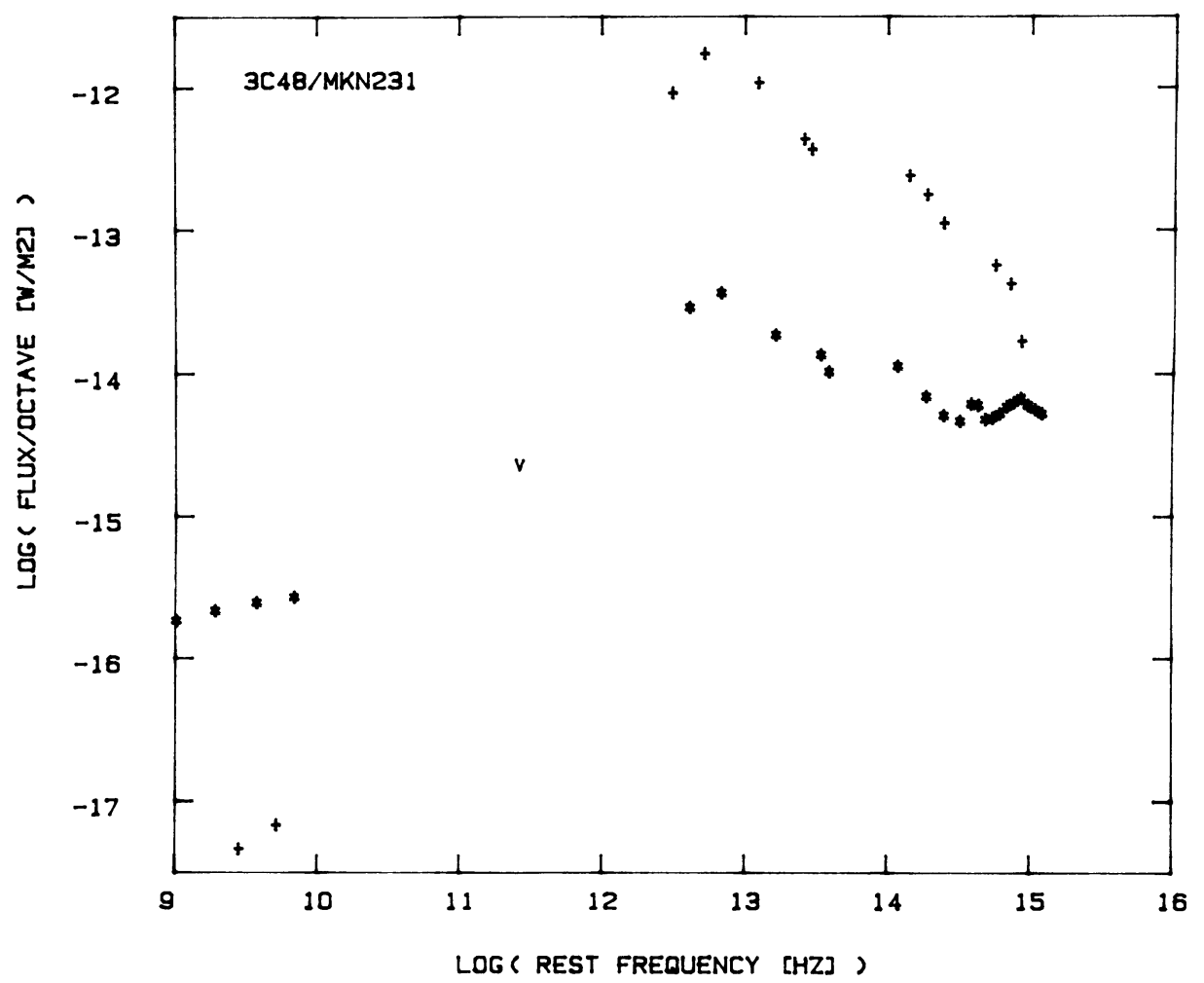

FIG. 2. - The continuum energy distributions of $3 \mathrm{C} 48\left(^{*}\right)$ and Mrk $231(+)$ are given. Bumps near $60 \mu \mathrm{m}\left(\right.$ frequency $\left.\approx 5 \times 10^{12} \mathrm{~Hz}\right)$, which are ascribed to thermal reradiation of heated dust grains, are clearly seen. Unless specifically referenced, the non-IRAS and non-near-infrared points in this and Figure 3 are as referenced by Véron-Cetty and Véron (1983). The near-infrared points were obtained at the Palomar Observatory. The radio points for 3C 48 are from Kellermann, Pauliny-Toth, and Williams (1969), while the millimeter limit is from Ennis, Neugebauer, and Werner (1982) and the visual observations are from Neugebauer et al. (1979).

kpc from the central source of $3 \mathrm{C} 48$ (Sandage and Miller 1966; Wampler et al. 1975). Wampler et al. estimate that the total mass in this nebulosity is about $10^{8} / N_{e} M_{\odot}$. Since $N_{e}$, the electron density, is likely to be at least $100 \mathrm{~cm}^{-3}$, again the far-infrared mass estimates are well in excess of those predicted for this region. If the dust is optically thick, the mass estimates from the far-infrared observations become larger.

There is evidence that the dust grains cannot exist as a closed shell around a single luminosity source, since such a shell must absorb the bulk of the luminosity of $3 C 48$, while there is no evidence for any extinction of the broad-line region. From the $\mathrm{H} \alpha: \mathrm{H} \beta$ ratio in $3 \mathrm{C} 48, E_{B-V}<0.06 \mathrm{mag}$ (Neugebauer et al. 1979), corresponding to a visual extinction of $A_{V}<0.2 \mathrm{mag}$. The infrared luminosity is about 6 times the luminosity observed in the visible. If the infrared radiation represents thermally reradiated visual/ultraviolet radiation from a shell around a single central luminosity source, then approximately one-seventh of the emitted luminosity is transmitted, i.e., the central source must be seen through $\sim 2 \mathrm{mag}$ of absorption at the wavelengths where the bulk of the luminosity is radiated from the central source. Although there is some uncertainty due to the unknown spectrum of the central source, the large discrepancy in the measured visual absorption from that inferred makes such a model highly implausible. In particular, any hypothesis that the dust grains are intimately located within or around the broad-line emission region becomes untenable.

Thus we suggest that the most likely location for the dust is in a galaxy surrounding the quasar. The implied mass of $>10^{10} M_{\odot}$ associated with the gas and dust if the gas-to-dust ratio is normal in $3 \mathrm{C} 48$, is typical of the mass in the interstellar medium in a galaxy. 3C 48 is visually accompanied by a luminous host galaxy. Gehren et al. (1984) have traced an irregular galaxy to a radial distance of $\sim 15^{\prime \prime}(160 \mathrm{kpc}$ at $3 \mathrm{C}$ 48 ) in the $r$ passband and measure it to be the "brightest host galaxy of any observed so far" with a luminosity of $\sim 5 \times 10^{11}$ $L_{\odot}$. Boroson and Oke (1982) have obtained spectra of the nebulosity $2^{\prime \prime}(20 \mathrm{kpc})$ from the point source and conclude it is dominated by hot stars. They further conclude that the galaxy is extremely luminous, $M_{V}=-23.4\left(L_{V} \approx 2 \times 10^{11} L_{\odot}\right)$. The observed infrared luminosity is comparable to that of Arp 220 (Soifer et al. 1984) as is the ratio of infrared to blue luminosity. Thus, while the observed luminosity is large, it is not unheard of for infrared galaxies.

It is seen from Figure 2 that the IRAS measurement at $12 \mu \mathrm{m}$ made with a $1^{\prime} \times 4^{\prime}$ diaphragm $(630 \times 2,500 \mathrm{kpc}$ at $3 \mathrm{C} 48$ ) exceeds that based on the ground-based observation at $10.6 \mu \mathrm{m}$ with a $5^{\prime \prime}$ diameter circular diaphragm $(50 \mathrm{kpc}$ at $3 \mathrm{C} 48$ ). This disparity may also be evidence for extended infrared emission from the galaxy surrounding $3 \mathrm{C} 48$. 

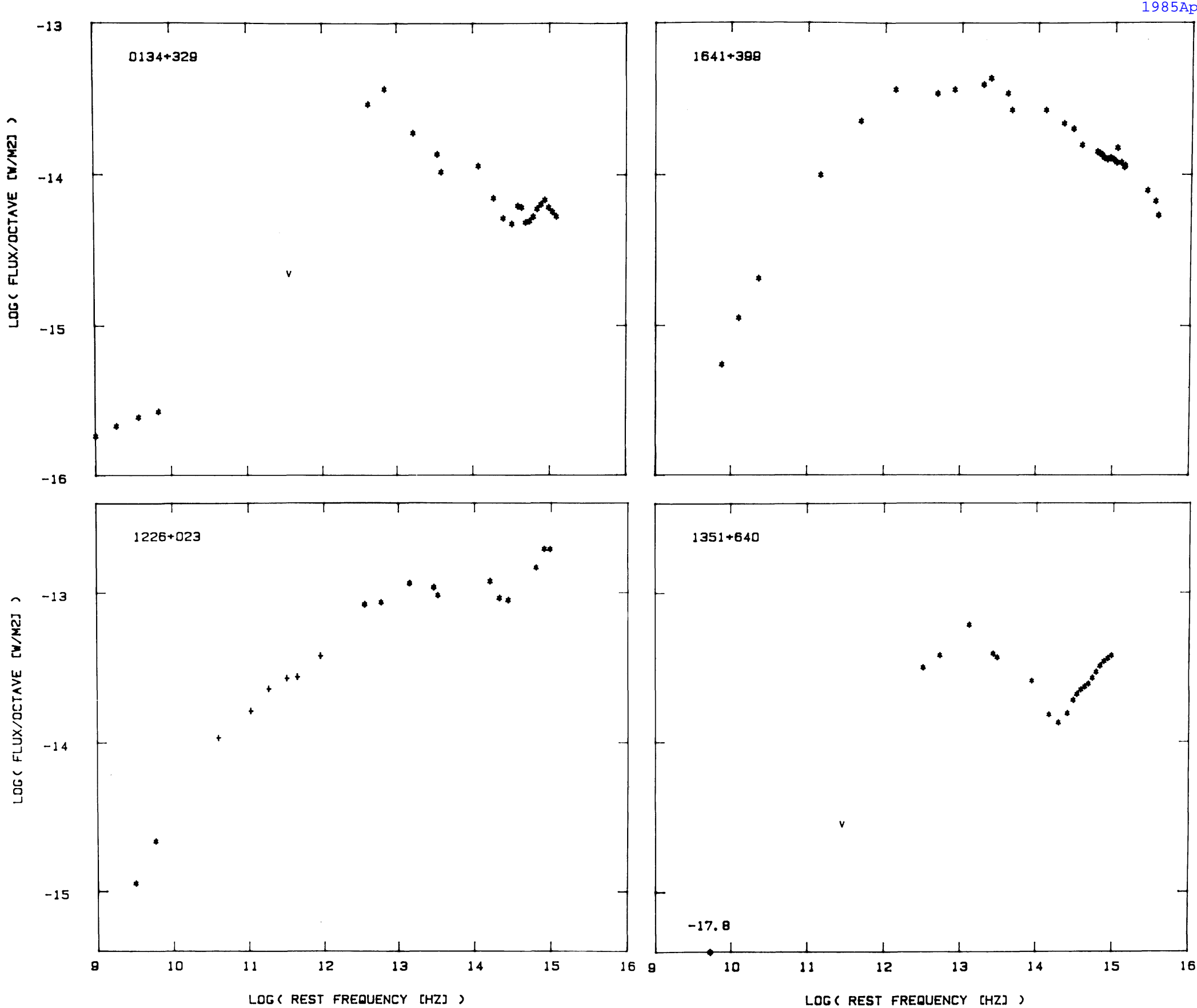

FIG. 3. - The continuum energy distributions for $3 \mathrm{C} 48(0134+329)$, 3C $273(1226+023), 3 \mathrm{C} 345(1641+399)$, and PG $1351+640$ are shown on the same scales. The $(+)$ in 3C 273 are from Clegg et al. (1983) and were obtained in 1982 April; the difference from a smooth curve probably reflects variability in the source. The measurements of 3C 345, including the IRAS and near-infrared points, were obtained in the 1983 April-May time period (Bregman et al. 1985). For 1351+640, the millimeter limit is from Ennis et al. (1982), the radio point is from Condon et al. (1981), and the visual observations are from Neugebauer et al. (1979). 
If, as suggested, this enormous luminosity, i.e., 6 times the quasar visual luminosity, is associated with the galaxy, its ultimate source and geometry is not well defined. If a single luminosity source is powering the system, then a special, though not unreasonable, viewing geometry must be invoked such that the galaxy subtends a sufficiently large solid angle to absorb the central luminosity while leaving the emission-line regions clear. An alternative explanation is to assume that the luminosity source is distributed throughout the galaxy, in which case the most plausible energy source for the emission is a truly enormous amount of active star formation. Such a situation is postulated, albeit on a much smaller scale, in NGC 1068 where an active nucleus and a ring of star formation are located in the same galaxy.

It is interesting to conjecture how universal in quasars is the presence of large amounts of infrared luminosity associated with thermal emission from dust. The continuum of the radio-quiet quasar PG $1351+640$ shown in Figure 3 gives an example of a quasar with peak emission at $25 \mu \mathrm{m}$. The luminosity in the $25 \mu \mathrm{m}$ peak is $\sim 4 \times 10^{11} L_{\odot}$, and the temperature derived from the $12-25 \mu \mathrm{m}$ flux densities is in the range of $190 \mathrm{~K}$. A similar $25 \mu \mathrm{m}$ peak, although of lower luminosity $\left(7 \times 10^{10} L_{\odot}\right)$ and stronger curvature between 12 and $60 \mu \mathrm{m}$ is present in the galaxy 3C 390.3 (Miley et al. 1984). The derived temperatures are comparable, but Miley et al. conclude the dust emission in 3C 390.3 arises within a $180 \mathrm{pc}$ core in that galaxy. Also, Miley, Neugebauer, and Soifer (1985) provide evidence that such mid-infrared thermal components are widespread in Seyfert 2 galaxies. Although the features ascribable to thermal reradiation from heated dust are not unique to $3 \mathrm{C} 48$, the effects of reradiation do not dominate the emission of the vast majority of quasars. A survey of the continua of quasars as observed by $I R A S$ is in preparation.

\section{CONCLUSIONS}

The infrared luminosity of $3 \mathrm{C} 48$ has been shown to be $\sim 5 \times 10^{12} L_{\odot}$. Although a previously undetected flat spectrum radio source cannot be excluded as the origin of this emission, we favor the hypothesis that it represents thermal reradiation from heated dust grains. It is argued that the luminosity originates outside the broad and narrow emissionline regions and that it most likely originates in a very luminous galaxy surrounding the quasar.

We thank all of our colleagues on the IRAS projectengineers, managers, and scientists-who have made these measurements possible. We especially thank the members of the subgroup on pointed observations of active galaxies and E. Young and E. Kopan for help in reducing the observations. We also thank our colleagues at Caltech, especially $\mathrm{K}$. Matthews and J. Elias, who have made many of the ground measurements over the course of several years. We thank $\mathrm{S}$. Phinney for pointing out the necessity for special geometry and $\mathrm{O}$. Neugebauer for discussions and providing G. N. the environment to write up these observations. The IRAS project is supported by NASA; ground-based infrared astronomy at Palomar is supported by a grant from the NSF.

\section{REFERENCES}

Baldwin, J. 1975, Ap. J., 201, 26

Boroson, T. A. and Oke, J. B. 1982, Nature, 296, 397.

Bregman, J., Glassgold, A. E., Huggins, P. J., and Kinney, A. L. 1985, Ap. J., 291, 505.

Clegg, P. E., et al. 1983, Ap. J., 273, 58

Condon, J. J. O'Dell, S. L., Puschell, J. J., and Stein, W. A. 1981, Ap. J. 246, 624 .

Draine, B. T. and Lee, H. M. 1984, Ap. J., 285, 89

Ennis, D. J., Neugebauer, G., and Werner, M. 1982, Ap. J., 262, 460

Explanatory Supplement to the IRAS Catalogs and Atlases. 1985 ed. C. A. Beichman, G. Neugebauer, H. J. Habing, P. E. Clegg, and T. J. Chester (Washington: US Government Printing Office) (IRAS Supplement).

Gehren, T., Fried, J., Wehinger, P. A., and Wyckoff, S. 1984, Ap. J., 278, 11.

Kellermann, K. I., Pauliny-Toth, I. I. K., and Williams P. J. S. 1969, Ap. $J ., 157,1$.

Miley, G. Neugebauer, G. Clegg, P. E., Harris, S., Rowan-Robinson, M., Soifer, B. T., and Young, E. 1984, Ap. J. (Letters), 278, L79.
Miley, G. K., Neugebauer, G., and Soifer, B. T. 1985, Ap. J. (Letters), 293, L11.

Neugebauer, G., Oke, J. B. Becklin, E. E., and Matthews, K. 1979, Ap. J., 230, 79 .

Neugebauer, G., et al. 1984, Ap. J. (Letters), 278, L1.

Sandage, A. R., and Miller, W. G. 1966, Ap. J., 144, 1238

Soifer, B. T., et al. 1984, Ap. J. (Letters), 283, L1

Soifer, B. T., et al. 1985 , in preparation

Véron-Cetty, M. P., and Véron, P. 1983, A Catalogue of Quasars and Active Nuclei (Munich: European Southern Observatory).

Wampler, E. J., Robinson, L. B., Burbidge, E. M., and Baldwin, J. A. 1975, Ap. J. (Letters), 198, L49.

Wilkinson, P. N., Spencer, R. E., Readhead, A. C. S., Pearson, T. J., and Simon, R. S. 1984, in IAU Symposium 110, VLBI and Compact Radio Sources, ed. R. Fanti, K. Kellermann, and G. Setti (Dordrecht: Reidel) p. 25 .

G. Neugebauer and B. T. SoIfer: Downs Laboratory of Physics 320-47, California Institute of Technology, Pasadena, CA 91125

G. Miley: Space Telescope Science Institute, Homewood Campus, The John Hopkins University, Baltimore, MD 21218 